\title{
WETTABILITY AND FRICCOHESITY OF EMULSIONS: COVID-19 KILLING ROBUST SCIENCE - A REVIEW
}

\section{Savita Sharma ${ }^{1 *}$ and Man Singh ${ }^{2}$}

${ }^{1}$ Haryana Education Department, Charkhi Dadri-127 306, Haryana, India. ${ }^{2}$ Chemistry Department, Central University of Gujrat, Gandhi Nagar, India.

\begin{abstract}
COVID-19 is currently a big threat to global health. However, no specific antiviral agent is available for its treatment yet. As per the vaccine is considered, there is a long way to go. So, the best treatment in present scenario are precaution and protection both. Covid-19 is menacing the whole of humanity and so the whole of humanity must fight back. The important tools used to fight against covid-19 are the two important properties of nanoemulsions -wettability and friccohesity. Nanoemulsions are considered as the colloidal monodispersion systems which are supposed to be thermodynamically and kinetically stable and composed of two or more immiscible liquid and emulsifying agents (surfactants and cosurfactant) mixed to form a single phase. Stable nanoemulsions are known to be investigated as efficient drug delivery systems. This review paper is formulated to provide understanding and consolidated information regarding different physicochemical properties of nanoemulsions like wettability, friccohesity and surface tension as a tool to fight against COVID19. This review paper also comprises a comparative study of various types of emulsions like soap, inorganic, and organic sanitizers role in fighting COVID19.
\end{abstract}

Keywords: Biocompatible, Friccohesity, Nanoemulsions, Surface tension and Wettability.

\section{INTRODUCTION}

Among various thermodynamically liquid formulations, the nanoemulsions are the most important formulations to enhance solubility and bioavailability of poorly water-soluble substances through micelles mechanism. They are referred as mini-emulsions or ultrafine emulsions of small droplet size(20$200 \mathrm{~nm}$ ) considered as thermodynamically and kinetically stable dispersions which consists of mixture of two or more immiscible liquids with water and oil which are stabilized by an interface film opening new domains of interfacial sciences. These consist of a surfactant and cosurfactant resulting in formation of a single phase tuned with few other hydrophilically and hydrophobically active ingredients. Several cationic, anionic, and nonionic surfactants with different properties have been used for formulating such industrially needed emulsions with needed surface charges. Among them, the routinely used are anionic surfactants (potassium laureate, sodium lauryl sulphate) cationic surfactants (quaternary ammonium halides), nonionic surfactant (sorbitan esters, polysorbate) and swifter ion surfactants as per need. Nanoemulsions which were formulated earlier were oils of variable saturation in water systems with average droplet diameter 50 to $1000 \mathrm{~nm}$. The nanoemulsions have been categorized into three different categories namely $\mathrm{o} / \mathrm{w}$ type, $\mathrm{w} / \mathrm{o}$, and biphasic or bicontinuous microdomains of water and oil are mutually interdispersed. Multiemulsions are considered as a category of nanoemulsions where both the oil/water and water/oil emulsions are present in one single phase system. For stabilization of two separate emulsions, hydrophilic and lipophilic surfactants are used for better and stable monodispersions. Stable nanoemulsions have 
many industrial advantages and some of them are noted categorically as. 1 . Increase rate of absorption number. 2. Reduced variance in absorption. 3. Protection of oil and water emulsions from oxidation and hydrolysis. 4. Transportation of lipophilic drugs after proper solubilization. 5. Efficient Aqueous doses form for water insoluble drugs. 6. Increased bioavailability of most of drugs. 7. Competence to incorporate lipophilic and hydrophilic drugs. 8. Efficient transport of drugs to increase efficacy with reduced side effects. 9. Serves as nontoxic and nonirritant transport medium for skin and mucous membrane delivery ${ }^{1} .10$. Support leaching out and coagulation of selected drugs or other molecules. Therefore, efficient, and authentic theories and studies are required to explore their potential mechanism which further could develop the novel nanoemulsions by partitioning the cohesive forces of the solvent or medium by generating an adhesive force with moderate frictional forces in a most mutual manner. Therefore, the cohesive and frictional forces develop localized oscillations, vibrations, rotational, translations motions of different orders within a single-phase liquid mixture as or monodispersed nanoemulsions. So, with nanoemulsion different energy states exist. Each constitutional unit with individualistic entropies develops spontaneity to partition and channelize a drug molecule to encapsulate it where the cohesive forces and the frictional forces become a great model for better drug transportation. Thereby engineering and operating these two forces could be a cutting edge for better nanoemulsion formulations and their applications. The operative product of the frictional and cohesive forces noted as a key to explore a desired stability of the targeted nanoemulsions is noted as friccohesity.

\section{Friccohesity}

Friccohesity lays a foundation of interacting or dynamic activities of surfactants, cosurfactant, stabilizers and other ingredients needed to formulate the nanoemulsion through their surface areas and surfaces charges with active participation. The friccohesity depicts molecular aggregate or disperse in specific solvent systems where the aggregation and dispersion are the backbone of chemical processes. Therefore, the friccohesity is considered as a dual force theory that deals with the frictional and cohesive type of forces and is determined with survismeter using Mansingh equation for determining the resultant molecular forces of single-phase liquid mixtures. Friccohesity is a dual theory and acts as an operator between cohesive forces and molecular forces among dissimilar molecules (MFDF). It depicts degree of dispersion, solubilization, degree of emulsification, degree of coagulation. Science of friccohesity define mutual operation of cohesive forces and molecular forces for better thermodynamics and kinetic stability.

Thereby, the friccohesity explains negative and positive deviations quantitatively, solvent structure breaking and making mechanism of the solute molecules based on the effective solvent binding in a most structured manner. If on adding solute the cohesive forces of the solvents undergo drastic decrease, i.e., the hydrogen bonds of the similar molecules are broken to the larger extent by the solute, then the cohesive force of the solvent is weakened. The weakening has been credited to an effective engagement of the solvent within 10 to $40 \mathrm{KJ}$ mol energy involvements. In this process, the solute molecules can dissolve in solvent in bulk medium like buffer, but the solute is not dissolved when there is no breaking of solvent hydrogen bonding. For instance, no cohesive forces are broken, as a result no dispersion or dissolution occurs when oil is added in water. The cohesive forces are changed into kinetic forces through frictional forces when cohesive forces are weakened. The solute particles move into the bulk of the solvent overcoming structural resistance of solvent particles. So, the cohesive forces are interchanged into frictional forces, and ultimately the product of cohesive force and frictional forces is referred as friccohesity which is measured by pendant drop numbers where the cohesive forces are measured and viscous flow times where frictional forces are measured. The survismeter with similar dimension is used in both measurements. It is seen that that such situation is complicated where giant molecules like proteins, supramolecules like calixarene, and smart molecules like TTDMM (trimesoyl tridimethyl malonate) dendrimer are involved. Because here many local interacting sites exist, and they interact within molecules as well as with the outer surface of the molecules ${ }^{2}$. Therefore, the friccohesity explores a hardcore host-guest chemistry of micelles and it becomes a most self-explanatory physicochemical state expressed as a friccohesity chemistry. Exceptionally the friccohesity chemistry elaborate each constitutional unit that develop localized cohesive or potential energy which interconnect with other operative unit or with vehicle or medium. The survismeter an analytical model explores, integrate, and optimize cohesive forces and adhesive or frictional forces on a mutual scale where the 
similar cohesive forces develop pendant drop numbers based on Fourier transform.

\section{Friccohesity Acting as Golden Theme in Nanoemulsion}

The friccohesity plays important role in case of ionic liquids (IL), liquid crystal (LC), triglycerides (TGL), and nanoemulsions (NEL) as these molecules' forms dispersion of hydrophobic particles in water phase and simultaneously their hydrophilic particles form different activities with water. In short, hydrophilic forces, enthalpic activity, and hydrophobic forces, and entropic activities act as the fundamental constituents for mutually partitioning the cohesive forces into adhesive forces with higher friccohesity. So, the hydrophobic part attacks hydrogen bonded water molecules with definite Brownian motion and it leads to develop frictional forces because its cohesive forces are weakened, so it can disperse only in structured water. Also, the hydrophilic particles lead to a formation of binding forces by disrupting structured water with weaker hydrophilic-hydrophilic transformations toward hydrophilic-water linkages or interfaces. Cohesive forces (CF) are largely weakened in hydrophilic water linkages that act as a favorable reason for increase in frictional forces also. So, the Brownian motions are favored by frictional forces in the form of hydrophobic tail and hydrophilic-water head weakens surfactant self-binding than to its binding with water molecules. When the Brownian motion is higher, a surfactant comes close to the surface and it gets settled on the surface and utilization of large amount of surface energy takes place. So, weakening of surface energy, which forms as a result of unbalanced molecular forces (UBMF) of the solvent particles, leads to development of molecular reorientation so as to optimize the forces according to their geometrical activities. Movement of larger surfactant molecules to surface is described as surface excess concentration per unit area of the surface. At the surface, two thermodynamically active domains work, hydrophilic and hydrophobic. When hydrophobic dominates, surface tension decreases with high pendant drop numbers when measured with survismeter. Simultaneously, frictional forces also become active and is increased when measured with survismeter in terms of viscous flow time. Generally, it is observed that surface tension decreases in case of non-electrolytes and also the viscosity is increased which acts as a proof for the fact that surfaces excess concentration is higher and bulk concentration is lower. This theory is defined by friccohesity because both the forces are active in such homogenized liquid mixtures and conductance is another data which plays a crucial role for defining the exact location, binding, and mobility of particles with higher forces or the lower forces ${ }^{2}$. The science of frictional and cohesive forces plays important role for association, dissociation, linkages, reorientation, and bond optimization of particles undergoing various changes. So, an optimization state of such forces is determined with $\sigma \propto$ t.n relation. Here $\sigma$ denotes frictional and cohesive forces denoted by $t$ and $n$ symbols, respectively. The Friccohesity $\sigma$ is measured with Mansingh equation as given below.

$$
\left.\sigma=\sigma_{0}\left[\left(\frac{t}{t_{0}} \pm \frac{B}{t}\right)\left(\frac{n}{n_{0}} \pm 0.0012(1-\rho)\right)\right] \quad \text { (ManSingh eqn. }\right)
$$

The $\mathrm{t}$ and $\mathrm{n}, \mathrm{t}_{0}$ and $\mathrm{n}_{0}$ denote viscous flow time and drop count for solution and solvent respectively, the $\mathrm{B} / \mathrm{t}$ is a kinetic energy correction, the 0.0012 air density and $(1-\rho)$ is buoyancy correction to drops. The $\rho$ denote density of the solution ${ }^{4}$.

\begin{abstract}
Wettability
Wettability is defined as the ability of a liquid to develop contact with the solid surface and it is controlled by the balance between the intermolecular interactions of adhesive type (liquid to surface) and cohesive type (liquid to liquid). Wettability is indicated as the presence of a liquid to be in contact with the solid surrounded by another fluid (liquid or gas). The wettability is a most desired property of the cosmetics, pesticides, soaps and detergents, nano thin film forming nanoemulsions, conducting nanothin films. Higher friccohesity values depicts higher wettability and uniform nanothin film formation. Farmers need a nanoemulsion of the Agri pesticides for spraying on crops where minimum inhibition concentration (MIC) is spayed on the leaves in a larger area with least consumption. Also, the higher friccohesity of textile dyes ensure uniform dyeing mechanism on the fabrics. The friccohesity is a green analytical concept because no density data are needed for its calculation because both the forces compensate the contributions of the density data.
\end{abstract}

Intermolecular Forces Define the Degree of Wettability 
Adhesive and cohesive forces between the solid and liquid determine the extent of wettability and its applicability. Cohesive forces operate in between the same type of molecules in a liquid or solid, and the molecules or the particles are pulled towards each other due to cohesive forces between the particles. Adhesion is defined as the interaction between unlike particles. The balance between these two forces, defines the degree of wettability by making the best use of the surface charges. For example, a drop of water on the solid substrate beads up, while ethanol on the same substrate tends to spread, this is because of the strong cohesive forces called hydrogen bonds that pull the particles towards each other. This infers that the ethanol with weaker cohesive forces has 21.55 $\mathrm{mN} / \mathrm{m}$ and spread more with higher wettability contrary to the water which has $71.97 \mathrm{mN} / \mathrm{m}$ surface tension. In this regards the friccohesity of ethanol is higher than of the water at the similar experimental conditions. And in case of ethanol, the Vander wall forces are responsible for cohesive interaction and these are much weaker than hydrogen bonds, so this causes ethanol to spread more easily on solid surface.

\section{Contact Angle as a Measure of Wettability}

Contact angle is a measure of extent of wettability. When a drop of liquid is placed on the solid surface, contact angle can be optically measured considering $90^{\circ}$ as a reference angle. Contact angle is described as a threshold value, when the contact angle is > $90^{\circ}$, the wettability is least noted as bad and when it is $<90^{\circ}$, a wettability is higher or good. A complete wetting is obtained when the contact angle is zero but in in a situation when contact angles are $<5^{0}$, the surface is considered, to be completely avoided and when water is used as a measuring liquid the surface is called hydrophobic.

Wettability of emulsions is an indicator of mutual and encounters of hydrophobic and hydrophilic chemical balances where in the case of normal micellar mixtures, the hydrophobic tail tend towards the central part or core. The water orients towards the outer layer of micelles which has ending of positive/negative nonionic head connected through electrostatic statics of water molecules. In such oriental mixtures, the friccohesity finds cohesive forces the water oriented around the peripheral layers and hydrophobic and hydrophobic forces inside the micelles. The strength of positionally placed molecules develop cosmetic ability. Such molecular interactions engineering leads to attain the thermodynamic, kinetic, and tentropic stability of resultant nanoemulsions.

\section{Measurement of Friccohesity and Wettability}

The physicochemical properties (PCPs) like surface tension, viscosity, interfacial tension (IFT), wetting coefficient do reflect different states of liquid mixtures obtained due to their structural reorientations accompanied by solute-solvent interactions. An ideal mixture has zero IMF, however, in case of non-ideal systems of the ionic and molecular components, there are interactions which are caused, due to, the structural constituents of medium with respect to the size and geometry of solute where friccohesity plays a critical role in understanding the mechanism behind the interaction. In past, it was impossible to measure the PCPs all at a time and with a single device but now it has been made possible by using a single device called Survismeter. The device not only saves $98 \%$ of the laboratory resources but is also an environment friendly and a smart device.

The researchers who are working in the field of solution chemistry are highly comfortable and find this device a very useful for measuring PCPs of their respective formulation. Previously, Ostwald viscometer, Ludwig Traube Stalagmometer and Ludwig Wilhelmy plate, Ubbelohde viscometer were used which require more investment and manpower along with an excess use of the laboratory resources. The Survismeter is a unique analytical model over the existing devices because it measures and tracks both the thermodynamic and transport (kinetic) properties together as a real spirit of the friccohesity. Hence Survismeter is an adequate answer for present experimental devices having more advantages with the minimum uses or consumption of the laboratory resources to explore interfacial sciences. Many researchers working on volatile liquids find it handy and secured for volatile liquids as the liquids are kept in a jacketed unit with no option of escaping of a sample molecule. It rules out all the possibilities of contamination and provides data with high degree of accuracy and precision. Survismeter has a vast area of applications in the field of pesticides, surfactants, emulsion, drug designing, dendrimers, nanoparticles, webdrimers, supramolecules, and many more which are the current hot topics in the field of the formulation chemistry ${ }^{31}$.

The surface tension (ST) and viscosity (V) of samples are measured using survismeter with 
high accuracy and precision. In one-time sample loading, many parameters can be determined. Sucking of the liquid is avoided because an improvised mechanical piston can do the job avoiding any health hazard as survismeter being a cost, energy and health protecting device. The survismeter is a most suited analytical equipment for academic, research and development laboratories, pharmaceutics, medical, engineering, hydrodynamic studies, automobile, aviation, environmental, municipal wastewater treatment endless and management fields. Survismeter allows accurate measurements with minimal discharge of polluting effluents in experiments for Surface Tension, Interfacial Tension, wetting coefficient, friccohesity, surface area, surface charges, activation energy, and Viscosity.

Survismeter is superior to various traditional devices because all these individual devices measure single property of a sample. As the survismeter $\mathrm{n}$-in-on ( $\mathrm{n}=$ multiple) in a single unit, so it saves washing reagents-chromic acid and acetone by $95 \%$ unlike tensiometers, Stalagmometer and viscometers when used for B. Tech, B. Pharm, B.Sc., M. Sc, M Tech, research, and others. And it is also used for quality of syrups, sol-gels, medicinal tablets, useful mixtures like coolants, dry-cleaning, atomizing mixtures like pesticides, spraying agents, disinfectants, cosmetics. Surface tension controls atomization of pesticides Aldrin and others for agricultural uses. Insecticides and pesticides require minimum surface tension for successful and economic process for saving large amounts because their excess use goes wastes along with poor quality formulations with excess amounts. It is a very simple and fascinating science ${ }^{4}$. This new research methodology termed as survismeter has been successful for the measurement of surface tension, viscosity, activation energy, interfacial tension, wetting coefficient, and friccohesity. Now, this new state of art is patented and commercialized by Borosil named as Borosil Mansingh Survismeter (BMS). Schematic illustration of survismeter is described in figure 1 given at the end ${ }^{2}$.

\section{Role of Soaps in Fighting Corona Virus}

This has been observed in many studies that soap is one of the effective ways to kill COVID-19 acting as a disinfectant. It dissolves fatty layer which coats corona viruses. Soap works very effectively on the SARS-CoV-2, the corona virus and almost all the viruses. It is due to, the fact, that soap is a self-assembled nanoparticle where the weakest link is the lipid (fatty) bilayer ${ }^{3}$. Thus, a higher friccohesity soap solution with higher percolating or wetting abilities dismantle the lipid bilayer of a cell.

Thordarson explains that viruses are mostly made up of three components as depicted in figure 2 :

- A nucleic acid genome (their genetic material: DNA or RNA).

- Protein, which encloses the nucleic acid and helps in viral replication inside a host body.

- A outer fatty layer made up of lipids.

The connections between these constituents form the structure of the virus, but these connections are weak as there are no covalent bonds which can provide a more stable structure. Thordarson says that the viral selfassembly of virus is based on weak "noncovalent" interactions between the proteins, RNA, and lipids. But together these constituents act like a Velcro, which is very hard to break up the self-assembled viral particle. Yet it is possible to break the particle with soap, which is very efficient in dissolving the lipid layer which surrounds the virus. It also breaks up all other weak bonds within the virus. Once this process ends, the virus effectively falls apart. Washing with water alone is less effective to shift the virus from the skin surface. Soap is composed of fat-like components called amphiphiles, which are the same as that of lipids found in the virus membrane. When soap approaches these fatty substances, it binds with these and disconnects them from the virus. It compels the virus to dispatch from the skin. Soap is composed of fat-like substances termed as amphiphiles, very similar to the lipids in the virus membrane. Thus, the soap molecules "compete" with lipids in the virus membrane. Viruses stick to the skin and get lodged in minuscule crinkles, that is why shifting them requires active handwashing. Therefore, it become essential to formulate the soap solutions of the higher friccohesity so it soaps molecules could easily approach the substrate for effective use.

Viruses get stick to oils which is present on our hands. But when we wash hands with soap, the soap contains surfactants which decrease surface tension and increases the wettability of water and results in the formation of micelles. So, in this way we are physically rubbing by friction and washing away virus to create an adequate rheology to dislocate the virus. Simultaneously friccohesity term also comes into action where the monodispersed soap molecules with higher kinetic energy and adequate tentropy surround the virus to disconnect it from its growing network. That is 
why surgeons scrub their hands very carefully before they go into an OT (operation theatre) 3 .

In formulations, the surface tension has been reduced so as to spread the water and wet the surfaces. The surfactants make water "wetter" by lowering the surface tension ${ }^{4}$. Hospital and community spread infections are increasing and create a serious public health problem worldwide ${ }^{5}$. Hands are the primary route for transmitting microbes and infections to the individuals ${ }^{6}$. Personal and hand hygiene is very important to prevent many communicable diseases. The word "hygiene" is derived from the ancient Greek goddess "Hygeia" which means "goddess of healing." The importance of hygiene is universally recognized, and is evidence based. It is a well observed fact that hand hygiene is crucial to prevent and minimize healthcare- associated infections ${ }^{7}$. The Centers for Disease Control and Prevention, the World Health Organization, and many other health experts promote hand hygiene as the single and most important measure to prevent hospital-acquired infections. Many studies have shown the importance of proper hand hygiene in controlling nosocomial infections $8,9,10,11,12$. It is estimated that at one time, more than 1.4 million people worldwide are suffering from infections acquired in hospitals. These nosocomial infections are in most cases, the result of poor hand hygiene ${ }^{13}$.

At present, washing hands with effective soap followed by applying hand antiseptics are two important hand hygiene method in clinical practice. Hand sanitizers efficiently increase the chances of maintaining the hands clean and aseptic. It is seen that the dendrimer like trimesoyl tridimethyl malonate - TTDMM, due to its functional cavity further partition the soap molecules which promote their monodispersion with stronger soap action.

\section{Limitations of Soap Hand Washing}

Scientific studies have proven that after hand washing, approximately $80 \%$ of individuals retain some pathogens on their hands ${ }^{14}$. Hand washing also along with cleaning removes body's own fatty acids from the skin, which may result in cracked skin that provides an entry portal for pathogens ${ }^{15,16}$. So, to overcome the limitations of plain hand washing, hand sanitizers were introduced which claim to be effective against pathogenic microorganisms as well as improve skin condition due to the addition of emollients into it ${ }^{17}$. These are the reason that the survismeter has been invented to tune the rheology with higher friccohesity utilizing the cohesive forces of the solvent in formulation on a scale of monodispersion.

\section{Role of Inorganic Hand Sanitizers in Fighting Corona Virus}

The chemical composition of most of the sanitizers is ethyl alcohol-70 to $90 \%$, hydrogen peroxide, glycerol, and distilled water. So, the main active ingredient of hand sanitizer is ethyl alcohol. The new corona virus is more sensitive to ethyl alcohol than SARS and MERS and it can be killed almost completely by ethanol of concentration as low as $30 \%$ as revealed by a joint study done by scientists from Germany and Switzerland. But World Health Organization (WHO) recommends 85\% alcoholic solutions, but some lower concentrations may do the work efficiently. The researchers said that the new corona virus seems to be more vulnerable to alcohol than other two deadly -SARS and MERS.

\section{Mechanism of Alcohol against Corona Virus}

The precise working mechanism of action of alcohol on the new corona virus needs more investigation, but a possible explanation can be attributed to its structure according to Pfaender. The corona virus also known as SARS-COV-2 has a similar structure to other corona viruses having a spiky membrane around its core. This membrane protects the core and allows the virus to bind to the human cells. Alcohol can initiate chemical reaction with biological materials like fatty acids on the membrane, but this does not destroy its gene as effectively as other disinfectant methods like heat and ultraviolet radiations. It can only disintegrate the protective envelope and destroys its capacity to infect and replicate.

According to Center for Disease Control (CDC) report and others, the most suitable explanation for the antimicrobial action of alcohol is the denaturation of proteins. The proteins tend to optimize in the homogenize liquid mixtures of the high friccohesity due to the robust interfacial activities of the nanoemulsion. This action is supported by the fact that absolute ethyl alcohol is a dehydrating agent and is less bactericidal than mixture of alcohols and water as proteins are denatured more rapidly in the presence of water ${ }^{18}$. The nanoemulsions of desired friccohesity and activation energy could also homogenize an alcohol vis-à-vis proteins on monodispersion mode. Methyl alcohol has the lowest bactericidal action of the alcohols, so it is rarely used in healthcare ${ }^{19}$. Ethyl alcohol of concentration 60 to $80 \%$ is very effective virucidal agent in activating all the lipophilic 
viruses (e.g. - herpes, vaccinia, and influenza virus) and many hydrophilic viruses (e.g.adenovirus, enter virus, rhinovirus, and rotavirus) but not hepatitis $A$ virus $(\mathrm{HAV})^{20}$ or polio virus ${ }^{21}$.

Isopropyl alcohol is not at all effective against the non-lipid enteroviruses, but it is fully affective against the lipid viruses. Some studies have also shown the capability of ethyl alcohol and isopropyl alcohol to inactivate the hepatitis $B$ virus (HBV) $)^{22,23}$ and the herbs virus $^{24}$ and ethyl alcohol to inactivate human immunodeficiency virus $(\mathrm{HIV})^{25}$, rotavirus, echovirus and astrovirus ${ }^{26}$.

Studies done by $\mathrm{Li}$ Zhang, a professor at school of public wealth in Nanjing Medical University, suggested that a liquor can be used as a disinfectant in emergencies when other products are not present. $\mathrm{He}$ also emphasized that rubbing with alcohol may not be very effective than washing hands with soap and water "physical removal can be safer and much cheaper". The disinfectant which has higher friccohesity with monodispersion of the molecules are most effective in action.

A study done by Japanese scientist Takaaki Nakaya published in the journal scientific reports in 2017 revealed that the interaction between alcohol and viruses in human body can be more sophisticated than in a test tube which decreases its effectiveness as a disinfectant. In this regard a measurement of friccohesity data for the formulations regarding their effectiveness could be a boon in such domain of the research because the environment of a test tube and the human differs in monodispersing thermodynamics. Therefore, this article advances the research in areas of destroying the viruses using disinfectants and fills up a gap of the understanding gathered out of this article visà-vis reported activities. The researchers said that the new coronavirus seems more vulnerable to alcohol than to other deadly viruses and SARS and MERS. Ethanol shows to deactivate SARS- COV-2 to viral infectivity. Therefore, the friccohesity chemistry could still explore new domain in understanding these critical interfacially vibrant sciences.

Considering the monodispersion dynamics obtained through the engineering of the friccohesity operator of medium and disinfectants molecules, the hand sanitizers have also been of use in reducing gastrointestinal illnesses in households, ${ }^{27}$ respiratory tract infections, and skin infections, ${ }^{28}$ in curbing absentee rates in elementary schools, ${ }^{29}$. These have also reduced illnesses in university dormitories ${ }^{30}$. Thereby, to reduce the infections in health care settings, alcohol-based hand sanitizers are recommended for the purpose of hand hygiene provided alcohol is also an effective constituent of a monodispersed nanoemulsion. Currently, a lot of hand sanitizers with variable degree of effectiveness are registered in National Agency for Food and Drugs Administration and Control. Where a physicochemical scenario on a scale of friccohesity profile for their thermodynamics, kinetic and tentropic stabilities could further be enhanced for better efficiency. Also, in outreach programs, screening procedures in routine practice, water scarcity areas, and bedside and chair-side clinical examination, hand sanitizers could be an alternative solution to achieve asepsis. The clinicians and common man face the problem in selecting the effective sanitizer.

Many products labelled as antimicrobial hand sanitizers are not so effective in decreasing bacterial counts on hands. The only reason is that their quality is not fitted in a monodispersion mode with higher friccohesity, so they coagulate with time or respond to visible light for redox activities. In this regard the higher friccohesity with monoadhesion of medium prevent such irregularities to maintain their higher effectiveness with time or during transportation. Their label claims of decreasing germs and harmful bacteria by $99.9 \%$, some studies infer a remarkable increase in concentration of bacteria in handprints impressed on agar plate after cleansing. There is still a need to verify the claims by the regulatory authorities to an enforce the good quality procedures.

The alcohol is the main active ingredient in alcoholic hand sanitizer to exert antimicrobial activity by causing protein denaturation, disruption of tissue membranes, and dissolution of various lipids. Alcohol has increasing effectiveness from $60 \%$ to $90 \%$ with 1-propanol being most effective followed by 2 propanol and finally by ethanol. Therefore, the quantize organic sanitizer could be a better alternative with natural ingredients extracted out of the fruit peels. Several studies have been conducted to estimate the antimicrobial activity of hand sanitizers alone, but restricted literatures assess the difference between many disinfectants and hand sanitizers contrary to the quantized organic sanitizers. Disinfectant chemical substances having an immediate and sustained activity destroys micro-organisms to a level required for hygienic and surgical indications. Sanitizing substances having an immediate activity which decreases the number of micro-organisms to a safer level to achieve the public health standards. Disinfectant contains a better form of alcohol (propanol) to gain more bacterial 
reduction compared to sanitizers (ethanol). Both can achieve bacterial reduction on contact in 15-30 sec time.

\section{Limitations of Alcohol-Based Sanitizers}

The alcohols being highly flammable are stored in cool well-ventilated area. These get evaporated rapidly making extended exposure time difficult to achieve unless the items are immersed for sterilization. The alcohol as a dehydrating agent makes the hands very dry. Alcoholic sanitizers are inflammable and easily catch fire, which can to accidents. This is the reason that the survismeter is used to measure the surface tension and viscosity or the friccohesity of the alcoholic sanitizers which are jacket in controlled pressure unit of the survismeter. The survismeter overcomes this limitation of characterization of alcoholic sanitizer. So, a use of quantized organic hand sanitizers is encouraged.

\section{Organic Hand Sanitizers}

Organic sanitizers are made from the phytochemical synthesized by plants like neem leaves, basil flowers basil leaves, mentha leaves, orange peel. Coriander, lime are the active constituents which are responsible for antimicrobial activity in organic and herbal hand sanitizers. Phytochemicals synthesized by plants lower the surface tension and increases the wettability. Lemon juice of chemicals act as the surfactant and the decrease the surface tension of water and nanoemulsions and micelles are formed so that sanitizer has the capability to dissolve the dirt particles in it. The organic hand sanitizers are biodegradable and can be used on vegetable and fruits to wash with it because surface tension of water is decreased by phytochemicals used in organic sanitizer. Recently Prof. Man Singh, Dean Central University of Gujarat along with his team synthesized a thermodynamically and kinetically stable quantized organic sanitizer which proves to be a remarkable tool to fight corona virus. He made high surface energy sanitizer using nerol, limonene, eucalyptol, geraniol, and some other phytochemicals obtained from many plants extracts. Plant extracts were used in a most stochiometric ratios through simple methods and were filtered through manifolding of clothes filters. No alcohol or synthetic surfactants were added to it.

\section{Mechanism of Organic Hand Sanitizer in Fighting Corona Virus}

As per the presentation: "Virus free veggies and fruits: Sanitizing in organic way" by Man Singh at Science Communication in the time of COVID19- Webinar on Citizen Science, this quantized organic sanitizer is a high energy monodispersed nanoemulsions that can enter anywhere in the cavity of even fruits and vegetables and develop the nanohydration spheres (NHS). The NHS have high kinetic energy, high surface area, entropy and least activation energy. It has a constructive entropy and so easily approach dust particles like corona virus and pesticides. Exact mechanism of the reaction is:

- First is to wet up the surface.

- Secondly it initiates washing and deterging of surface starts.

Geraniol is a phytochemical present in citrus family acts as a molecular tweezers and it is wholly dispersed in sanitizer and its structural constituents come closer to corona virus protein and RNA, so it tweezes out and develops mechanism to detach it from the surface. Fundamental principle is that until the sanitizing molecule and virus come at a working distance, nothing happens. Wetting the surface here means, it approaches the virus and comes at a working distance to interact and has high potential energy, so a sanitizer starts functioning and this denatures the protein of corona virus. Proteins are undissolved in water but when phytochemicals are present, they have hydrophobicity and hydrophilicity, and proteins are hydrophobic in nature. At this stage of coordination and optimization, corona may be defunct. There is least interfacial energy between sanitizer molecule and surface, so there is excellent wetting, washing, and detaching of the corona virus.

There are basically two types of surfaces, uniform surfaces in which washing is uniform and second is non uniform surfaces, when some unknown activities are attached or initiated on surfaces, the quantized organic sanitizer acts in a different mode because potential energy, entropy, and wettability changes. The quantized organic sanitizer behaves like a Newtonian fluid which is highly stable on thermodynamic, kinetic, and tentropy scale and do not degrade or structurally changes with time. The quantized organic sanitizer has lowest cohesive forces with higher friccohesity. These sanitizing molecules develop least interfacial energy between two molecules, so these provide highest wettability with lowest interfacial energy.

\section{Advantages of Organic Sanitizers Conventional or inorganic sanitizers damage surfaces}


- Conventional or inorganic sanitizers mainly uses sodium hypochlorite $(\mathrm{NaOCl})$ and alcohol.

- Sodium hypochlorite used in normal sanitizers corrodes the surface due to ionic species.

- $70 \%$ ethyl alcohol has high osmotic pressure and attracts water from surface, so it acts as dehydrating agent and dries the skin.

\section{Quantized Organic Sanitizers}

- Quantized Organic sanitizers are nanohydration spheres and molecules remains monodispersed which neither corrodes nor dehydrates the surface.

- Many phytochemicals like limonene and eugenol are present in nanohydration forms, they develop multiple energy pockets, and these pockets are not of same energy. There is spontaneity in quantized organic sanitizers, it does not aggregate, and clusters wash out the virus efficiently.

- Quantized Organic sanitizer entangles the corona virus.

- Quantized Organic sanitizers lead to virus free veggies, fruits, and other eatables. They enter the microcavities of veggies and fruits and thin film is formed.

- Quantized Organic sanitizers are 100\% safe and biocompatible, so can act as a major preventive tool against corona virus and some other infections also.

\section{CONCLUSION}

After reviewing all the possibilities, we reached to the conclusion that the soap acts as the best preventive measure in which both wettability and friccohesity plays a major role in fighting corona virus through the most vibrant interfaces. But more frequent washing with soap can let the skin very dry, in that case herbal soaps can do the work more efficiently than ordinary soaps. As per the sanitizers are considered, best solution of the problem is to use quantized organic sanitizers instead of inorganic sanitizers. I appreciate Prof. Man Singh, who took great initiative to come forward and lead the way during the crucial scenario of COVID19 to innovate amazing formulation of quantized organic sanitizer, in which wettability and friccohesity proves to be COVID19 killing robust science. This innovation proves a new proverb which is consistent during this time- "Crisis is the mother of invention". Survismeter which was innovated by Prof. Man Singh as illustrated earlier in the review is the green, clean and sample is recycled in its measuring units, it does not come in contact of environment. Survismeter is also handy, cost effective kit to be kept at kitchen for safety reasons. So, more and more use of quantized organic sanitizers should be encouraged as a preventive tool to fight COVID19. A lot of further research work can be done in synthesizing many more quantized organic sanitizers which are cheap, biocompatible and environment friendly too.

\section{ACKNOWLEDGEMENT}

Author thanks Prof. Man Singh, Dean Central University of Gujrat for providing valuable support, insight and guidance in formulating this review paper. 


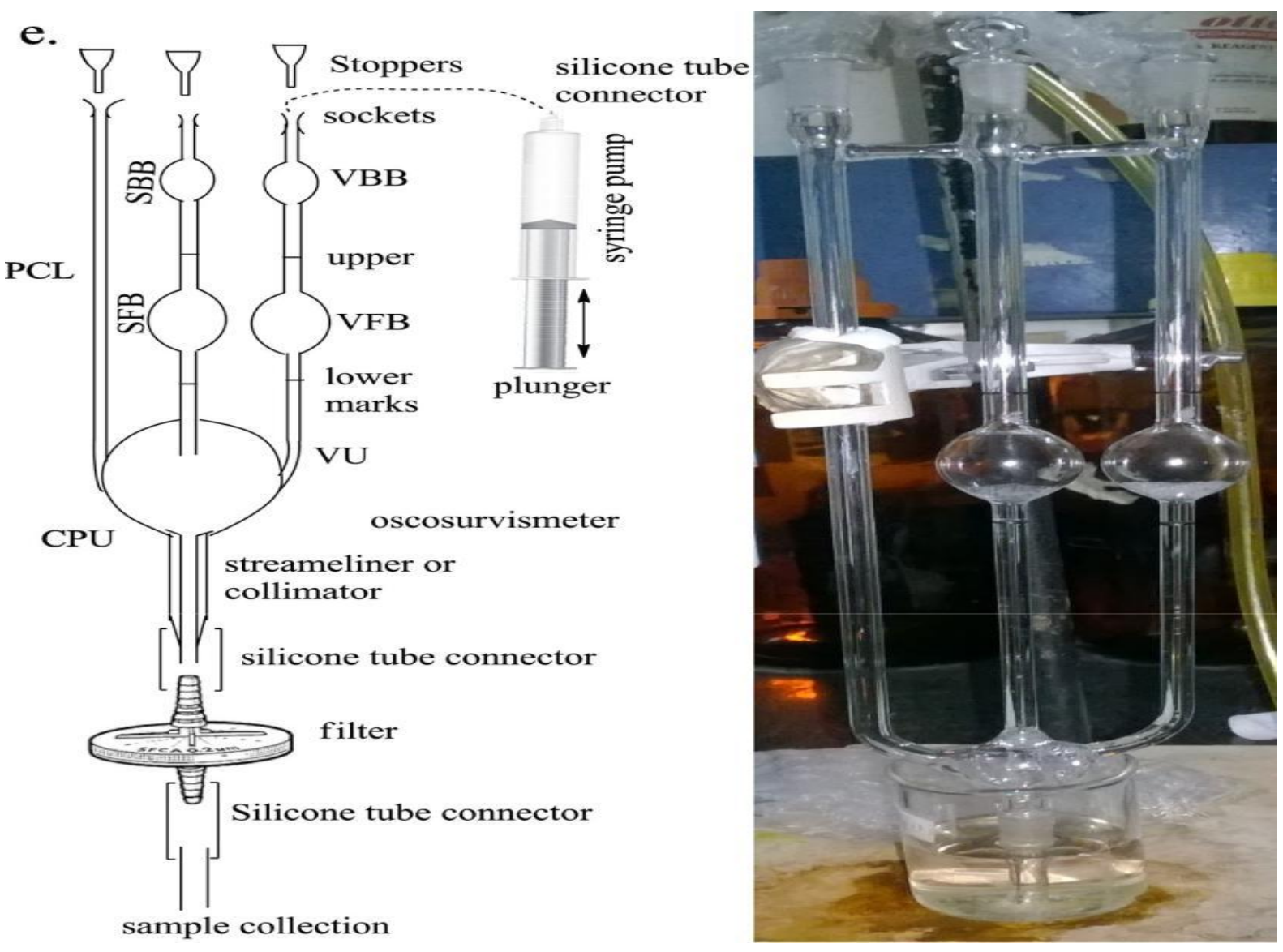

Fig. 1: Survismeter

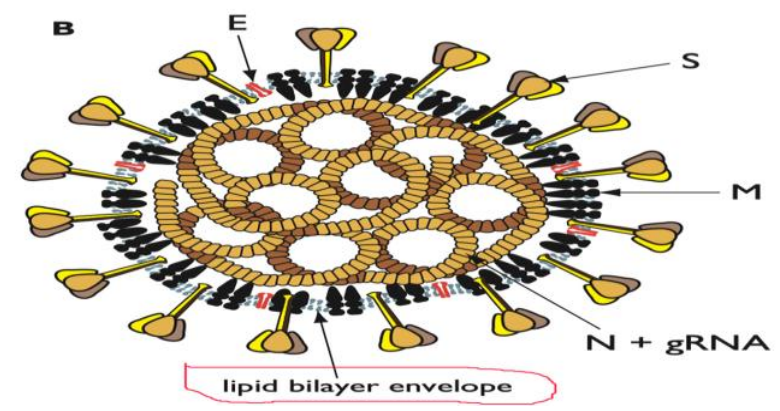

Fig. 2: Figure of Corona Virus. 


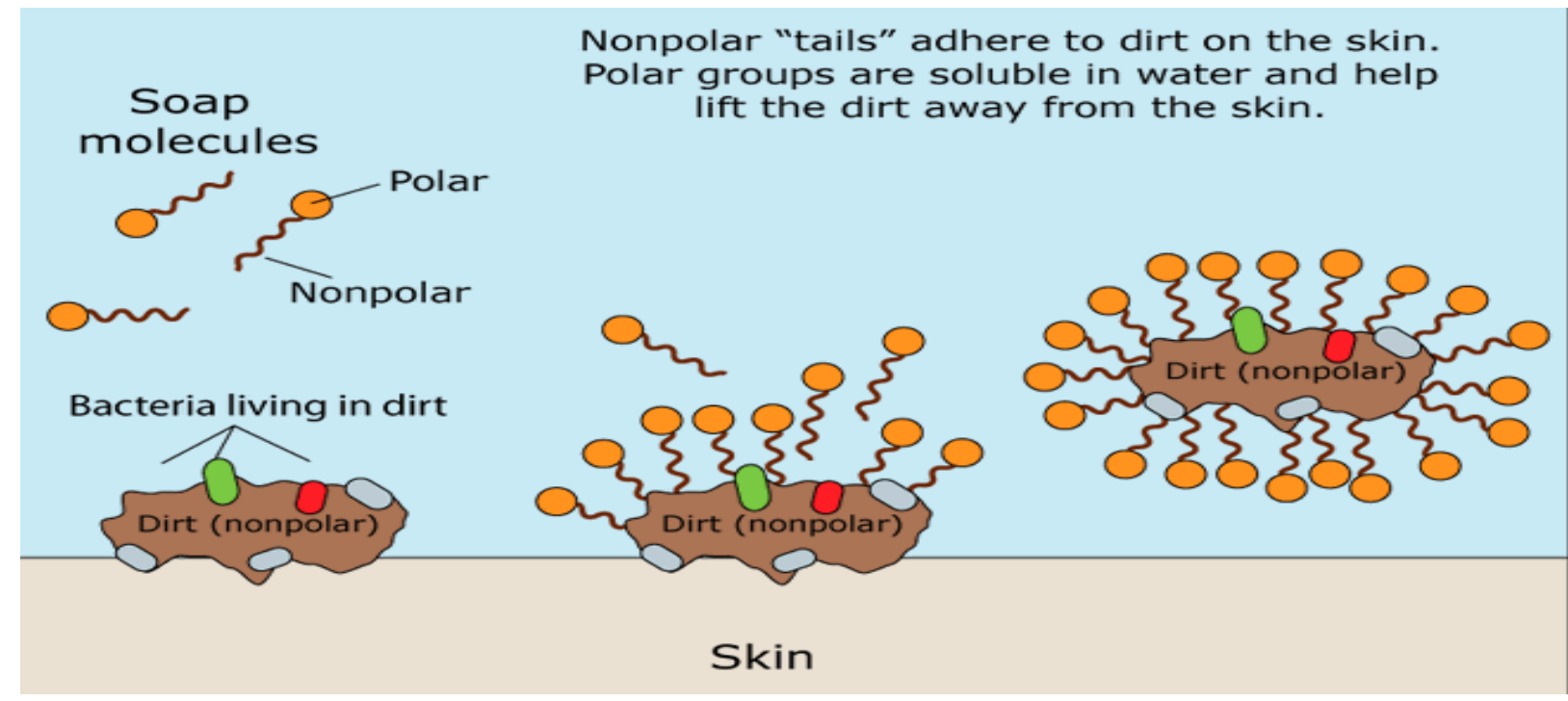

Fig. 3: Action of soap molecules on dirt particles.

\section{REFERENCES}

1. Gurpreet K and Singh SK. Review of nanoemulsion formulation and characterization techniques. Indian $\mathrm{J}$ Pharma Sci. 2018;80(5):781.

2. Singh M. Friccohesity and Tentropy. New Models of Molecular Sciences. http://dx.doi.org/10.5772/62240.

3. Covid-19 experts explains what soap does to SARS-COV2 Virus. https:// newsnetwork.mayoclinic.org.

4. Singh M. The Survismeter- A Green Technology in Service. Clean Technology. 2009. www.ct-si.org. ISBN 978-1-4398-1787-2.

5. Hassan AO, Hassan RO, Muhibi MA and Adebimpe WO. A survey of Enterobacteriaceae in hospital and community acquired infections among adults in a tertiary health institution in Southwestern Nigeria. Afr J Microbiol Res. 2012;6:5162. [Google Scholar].

6. Mondal $S$ and Kolhapure SA. Evaluation of the antimicrobial efficacy and safety of pure hands herbal hand sanitizer in hand hygiene and on inanimate objects. Antiseptic. 2004;101:55. [Google Scholar].

7. Pratt RJ and Pellowe C. The epic project: Developing national evidencebased guidelines for preventing healthcare associated infections. Phase 1: Guidelines for preventing hospital-acquired infections. Department of Health (England) $\mathrm{J}$ Hosp Infect. 2001;47(Suppl 1):S3-82. [PubMed] [Google Scholar].
8. Centers for Disease Control and Prevention. Guideline for Hand hygiene in health-care settings: Recommendations of the Healthcare Infection Control Practices Advisory Committee and the HICPAC/SHEA/APIC/IDSA hand hygiene task force. MMWR. 2002;51:1. [PubMed] [Google Scholar].

9. World Health Organization. WHO/IER/PSP/2009/01. Geneva, Switzerland: World Health Organization ; WHO Guidelines in Hand Hygiene in Health Care. 2009. [Google Scholar]

10. Erasmus V, Kuperus MN, Richardus $\mathrm{JH}$, Vos MC, Oenema $A$ and Van Beeck EF. Improving hand hygiene behaviour of nurses using action planning: A pilot study in the intensive care unit and surgical ward. J Hosp Infect. 2010;76:161(2010). [PubMed] [Google Scholar].

11. Pittet $D$, Allegranzi $B$ and Byc J. The World Health Organization guidelines on hand hygiene in health care and their consensus recommendations. Infect Control Hosp Epidemiol. 2009; 30:611. [PubMed] [Google Scholar].

12. Son C, Chuck T, Childers T, Usiak S, Dowling $\mathrm{M}$ and Andiel C. Practically speaking: Rethinking hand hygiene improvement programs in health care settings. Am J Infect Control. 2011;39:716(2011). [PubMed] [Google Scholar]

13. Madan K, Prashar N and Thakral S. Comparative evaluation of efficacy of 
alcoholic vs. non-alcoholic hand sanitizers. Int J Life Sci Biotechnol Pharma Res. 2012;1:173. [Google Scholar]

14. Tambekar D, Shirsat SD, Suradkar SB, Rajankar PN and Banginwar YS. Prevention of transmission of infectious disease: Studies on hand hygiene in healthcare among students. Cont J Biomed Sci. 2007;1:6. [Google Scholar]

15. Larson EL, Hughes CA, Pyrek JD, Sparks SM, Cagatay EU and Bartkus JM. Changes in bacterial flora associated with skin damage on hands of health care personnel. Am J Infect Control. 1998;26:513(1998). [PubMed] [Google Scholar].

16. Winnefeld M, Richard MA, Drancourt $M$ and Grob JJ. Skin tolerance and effectiveness of two hand decontamination procedures in everyday hospital use. Br J Dermatol. 2000;143:546. [PubMed] [Google Scholar]

17. Lauharanta J, Ojajärvi J, Sarna S and Makela P. Prevention of dryness and eczema of the hands of hospital staff by emulsion cleansing instead of washing with soap. J Hosp Infect. 1991;17:207. [PubMed] [Google Scholar].

18. Guidelines for disinfection and sterlization in healthcare facilities. Center for Disease Control \& Prevention (CDC) Report. 2008.

19. Tilley FW and Schaffer JM. Relation between the chemical constitution and germicidal activity of the monohydric alcohols and phenols. J. Bacteriol. 1926;12:303.

20. Mbithi JN, Springthorpe VS and Satter SA. Chemical disinfection of hepatitis $A$ virus on enviornmental surfaces. Appl Enviorn Microbioi. 1990;56:3601.

21. Tyler R and Ayliffe GA. A surface test for virucidal activity of disinfectants: preliminary study with herpes virus. J. Hosp.Infect. 1990;15:339.

22. Bond WW, Favero MS, Ptersen NJ and Ebert JW. Inactivation of hepatitis $B$ virus by immediate to high level disinfectant chemicals. J Clin Microbiol. 1983;18:535.
23. Kobayashi $\mathrm{H}$ and Tsuzuki $\mathrm{M}$. The effect of disinfectants and heat on hepatitis virus. J Hosp Infect. 1984;5:93.

24. Tyler R and Ayliffe GA. A surface test for virucidal activity of disinfectants: preliminary study with herpes virus. J Hosp Infect. 1987;9:22.

25. Martin LS, Mc Dougal JS and Loskoski SL. Disinfection and inactivation of the human $T$ lymphotropic virus type///lymphadenopathy- associated virus. J Infect Dis. 1985; 152:400.

26. Kurtz JB, Lec TW, Parsons AJ. The action of alcohol on rotavirus, astrovirus and enterovirus. J Hosp Infect Dis. 1985;152:400.

27. Sandora TJ, Taveras EM, Shih MC, Resnick EA, Lee GM and RossDegnan D. Abstracts of the 42nd annual meeting of the Infectious Disease Society of America; Boston, Massachusetts; 30 September, 3 October 2004. Alexandria (VA): Infectious Disease Society of America; 2004. Hand sanitizer reduces illness transmission in the home. [abstract 106] [Google Scholar].

28. Bloomfield SF. The effectiveness of hand hygiene procedures in reducing the risks of infections in home and community settings including hand washing and alcohol-based hand sanitizers. Am J Infect Control. 2007;35:27. [Google Scholar

29. Hammond B, Ali Y, Fendler E, Dolan $M$ and Donovan S. Effect of hand sanitizer use on elementary school absenteeism. Am J Infect Control. 2000;28(5):340. [PubMed] [Google Scholar]

30. White C, Kolble R, Carlson R, Lipson $\mathrm{N}$, Dolan $\mathrm{M}$ and Ali $\mathrm{Y}$. The effect of hand hygiene on illness rate among students in university residence halls. Am J Infect Cont.

31. Singh M. Physicochemical properties of Liquids: Friccohesity and Survismeter. http://ezinearticles.com/expert/Man_ Singh/233436. 